\title{
Research Square \\ On nature of determining displacement from sole knowledge of self interferometric perturbed phase
}

Junaid Khan ( $\nabla$ jkhan.bee15seecs@seecs.edu.pk)

\section{Research}

Keywords: Self mixing interferometery,phase equation,L2 triangular ineqaulity, Riesz-Thorin theorem, convolution theorem, sine half and double angle identity

Posted Date: May 10th, 2020

DOI: https://doi.org/10.21203/rs.3.rs-27184/v1

License: (c) (i) This work is licensed under a Creative Commons Attribution 4.0 International License. Read Full License 


\title{
On nature of determining displacement from sole knowledge of self interferometric perturbed phase
}

\author{
Junaid Iqbal Khan
}

Received: date / Accepted: date

\begin{abstract}
While self mixing interferometry(SMI) has proven to be suitable for displacement measurement and other sensing applications,its characteristic self mixing signal shape is strongly governed by the non-linear phase equation which forms relation between perturbed and unperturbed phase of self mixing laser.Therefore, while it is desirable for robust estimation of displacement of moving target, the algorithms to achieve this must have an objective direction which can be achieved by understanding the characteristic of extracting knowledge of perturbed phase from unperturbed phase. Therefore, this paper tends to deduce results from the self mixing interferometry(SMI) phase equation, by showing the impossibility of analytically deriving perturbed phase as continuous function of unperturbed phase either through finite or infinite substitutions. Therefore we never get exact displacement just by sole knowledge of perturbed phase. Moreover, it has theoretically shown that the action of practically deriving unperturbed phase from perturbed phase becomes a filtering process in spectral domain followed by perturbative or homotopical deformation.
\end{abstract}

\section{Keywords}

Self mixing interferometery,phase equation, $L_{2}$ triangular ineqaulity, Riesz-Thorin theorem, convolution theorem,sine half and double angle identity

Junaid Iqbal Khan

National University of Sciences and Technology, Pakistan

Tel.: +92-314-5251237

E-mail: jkhan.bee15seecs@seecs.edu,pk 


\section{Introduction}

Laser diode(LD) based Self-Mixing (SM) or optical feedback interferometry is an attractive sensing scheme due to the compact, self-aligned, and low-cost nature of the SM sensor[10]. A basis process of self mixing phenomenon for

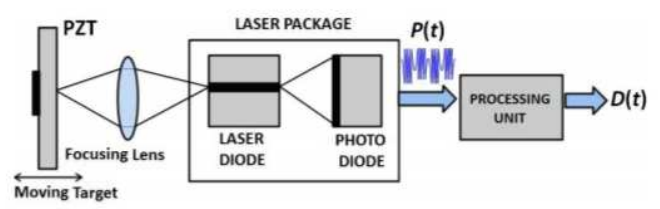

Fig. 1 Schematic diagram of self-mixing laser sensor for displacement measurement requiring only a laser package and a lens. A piezoelectric transducer (PZT) has been used as remote target. Variations in the optical output power $\mathrm{P}(\mathrm{t})$ are processed by a computing unit in order to retrieve the target displacement $\mathrm{D}(\mathrm{t})$

displacement measurement is shown in figure(1). An SM sensor is much simpler than conventional interferometers because many optical elements,such as the beam-splitter, reference mirror and external photo-detector are not required.Thus, with a simply constructed opto-electronic system, smart laser non-contact sensors have been developed using SM [9] with high precision, [3]. 2]. Model of optical feedback by Langand Kobayashi [6], shows that displacement retrieval from a weak feedback regime SM signal was achieved by unwrapping the laser feedback phase. It used pre-calibrated values of two fundamental SM parameters: line-width enhancement factor $\alpha$ and optical feedback coupling factor $\mathrm{C}$. The method is claimed to have measurement error of $<50 \mathrm{~nm}$ in displacement reconstruction for laser wavelength of 673 $\mathrm{nm}$, resulting in an accuracy of about $\frac{\lambda}{13}$. Further improvement was achieved by an auto-adaptive SM retrieval algorithm, called as the phase unwrapping method(PUM), for moderate feedback regime [2]. It consists of two major steps: 1) rough estimation of laser phase under feedback $\phi_{t}$ and 2) joint estimation of $\mathrm{C}, \theta=\arctan (\alpha)+\phi_{o}$, and displacement $\mathrm{D}(\mathrm{t})$, where $\phi_{o}$ represents the initial laser phase under feedback. The proposed method increases the accuracy of the SM sensor to $\frac{\lambda}{16}$. The root of the this research was tackeled in [7], where behavioral model of a self-mixing laser diode sensor was presented to simplify the solutions to non-linear self interferometric phase equation and thus modelling the basis of all algorithms by means of piecewise analysis on cases of C. Moreover, in [10], TFSP method was introduced where perturbed phase was estimated from unperturbed phase thus removing the need for estimation of parameters of phase equation, by means of Fourier series but to my understanding, the logic of generally connecting Fourier coefficents of perturbed phase and unperturbed phase remained unproven. Therefore analysis has been performed on this paper in order to rigorously deduce the spectral 
property of perturbed phase as compared to unperturbed phase, along with providing theoretical background on deriving them in time domain.

\section{Main theory of Self Mixing Interferometry}

Laser beam is generated in the optical cavity of laser diode and a portion of the laser beam is back-scattered from target and re-enters the active laser cavity. Let $\mathrm{D}(\mathrm{t})$ represent the instantaneous distance between the LD driven by a constant injection current and a remote surface which back-scatters a small amount of optical power back into the LD cavity[7]. When this optical feedback phenomenon occurs, the laser wavelength is no longer the constant $\lambda_{o}$ but is slightly modified and becomes a function of time $\lambda_{f}(t)$ when $\mathrm{D}(\mathrm{t})$ varies. The wavelength fluctuations can be found by solving the phase equation,

$$
x_{o}(t)=x_{f}(t)+C \sin \left(x_{f}(t)+\arctan (\alpha)\right)
$$

$x_{o}$ and $x_{f}$ are referred as perturbed and unperturbed phase respectively. $\mathrm{C}$ is the optical feedback coupling factor and $\alpha$ is the line enhancement factor. $x_{o}$ and $x_{f}$ can be represented as,

$$
\begin{aligned}
& x_{o}(t)=2 \pi \nu_{o}(t) \tau(t) \\
& x_{f}(t)=2 \pi \nu_{f}(t) \tau(t)
\end{aligned}
$$

where $\tau(t)=2 D(t) / c$ is the round trip time, with $\mathrm{c}$ as speed of light. $\nu_{f}(t)$ and $\nu_{o}(t)$ represents optical frequencies with and without feedback. As $\mathrm{C}$ increases from zero, the laser operates into five different regimes.Generally, (self mixing)SM sensing is performed under weak feedback regime $(C<1)$, moderate feedback regime $(1<C<4.6)$, or strong feedback regime $(C>4.6)$. However, moderate feedback regime is usually preferred over others as the apparently simple saw-tooth shaped SM fringes belonging to such a regime [1] intrinsically provide motion direction indication and require simplified SM fringe detection processing (while both tasks are difficult to achieve for weak feedback regime). Laser feedback output optical power(LDOOP) $\mathrm{P}(\mathrm{t})$ depends on the SM phenomenon and written as:

$$
P(t)=P_{o}\left[1+m \cos \left(x_{f}(t)\right)\right]
$$

where $P_{o}$ is the power emitted by the free running state LD and $\mathrm{m}$ is the modulation index.From above, perturbed phase $x_{f}$ can be extracted, which can be used to calculate unperturbed phase $x_{o}$ and then displacement $\mathrm{D}(\mathrm{t})$ of the vibrating target by,

$$
D(t)=\frac{\lambda_{o} x_{o}}{4 \pi}
$$




\section{Methods}

This paper inductively provides the nature of algorithms for estimation of displacement from sole knowledge of perturbed phase of SMI phase equation(1), while parameters $\mathrm{C}$ and $\alpha$ might be uncertain, by proving certain results in time and frequency domain through mathematical analysis. These results were tested through simulations

\section{Analysis}

5.1 Impossibility of derivation of perturbed phase as continuous function of unperturbed phase from SMI phase equation

Consider the SMI phase equation,

$$
x_{o}=x_{f}+C \sin \left(x_{f}+\arctan (\alpha)\right)
$$

Putting $\theta=\arctan (\alpha)$,

$$
x_{f}-x_{o}=-C \sin \left(x_{f}+\theta\right)
$$

Suppose that $x_{o}$ is and invertible continuous function of $x_{f}, x_{o}=G\left(x_{f}\right)$, then $x_{f}=G^{-1}\left(x_{o}\right)=H\left(x_{o}\right)$. If after following steps solutions obtained are not extraneous,then our assumption is correct.

using sine double angle identity,

$$
\begin{gathered}
\sin (2 x)=2 \sin (x) \cos (x) \\
\sin (x)=2 \sin (x / 2) \cos (x / 2) \\
\sin (x)=2 \sin (x / 2) \sin (\pi / 2-x / 2)
\end{gathered}
$$

we get,

$$
x_{f}-x_{o}=-2 C \sin \left(\frac{x_{f}+\theta}{2}\right) \sin \left(\frac{\pi}{2}-\frac{x_{f}+\theta}{2}\right)
$$

Bringing the arguments of sine to form of $(f+\theta)$

$$
x_{f}-x_{o}=-2 C \sin \left(\frac{x_{f}-\theta}{2}+\theta\right) \sin \left(\frac{\pi}{2}-\frac{x_{f}}{2}-\frac{3 \theta}{2}+\theta\right)
$$

Using the original phase equation, we can make substitutions as,

$$
x_{f}-x_{o}=-2 C\left(\frac{x_{o}-\frac{x_{f}-\theta}{2}}{C}\right)\left(\frac{x_{o}-\frac{\pi-x_{f}-3 \theta}{2}}{C}\right)
$$

The above forms a quadratic equation, whose solutions of $x_{f}$ in terms of $x_{o}$ appear as,

$x_{f}=\frac{1}{2}\left(2 C+\pi-2 \theta \pm \sqrt{4 C^{2}+4 C \pi+\pi^{2}-8 C \theta-8 \pi \theta+16 \theta^{2}-8 C x_{o}-8 \pi x_{o}+32 \theta x_{o}+16 x_{o}^{2}}\right)$

Firstly,these solutions are obviously extraneous as, when put back in (7), we do not get any feasible relation, as no identity relation of sine function interacting with under root leads to an algebraic equation. Therefore, all analytic 


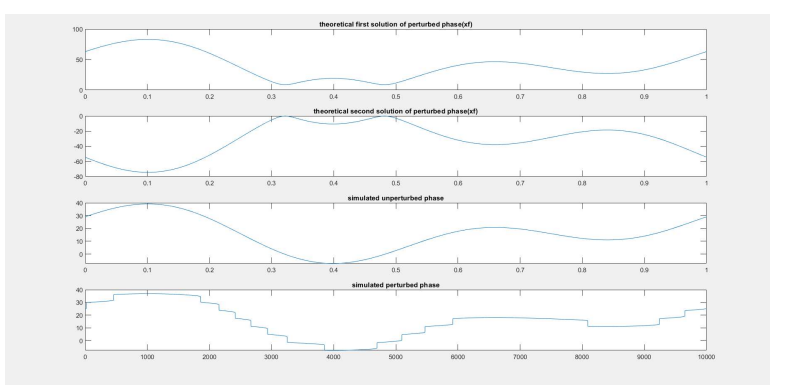

Fig. 2 First two plots represent theoretical perturbed phase solutions, last two plots represent simulated unperturbed and corresponding perturbed phase with $\mathrm{C}=5, \alpha=5$

solutions are extraneous.

On simulating results by figure(2), the simulated perturbed phase does not match theoretical perturbed phase solutions. Therefore, all the solutions derived are extraneous and thus no mapping representing $x_{f}$ as closed function of $x_{o}$ can satisfy phase equation, as the inverse does not exist and our assumption becomes wrong.

5.2 Impossibility of achieving perturbed phase from unperturbed phase through infinite substitutions from SMI phase equation

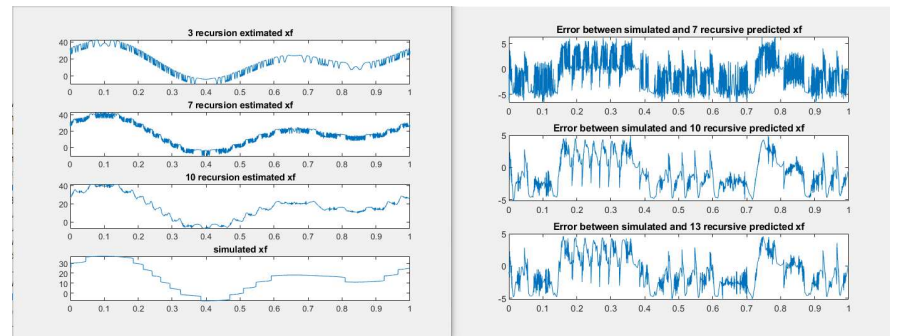

Fig. 3 left side first three plots represent theoretical perturbed phase solutions for 3,7 and 10 recursions,left last plot represents simulated perturbed phase,right side three plots represent error between perturbed phase and 7,10,13 recursive perturbed phase from top to bottom, with $\mathrm{C}=5, \alpha=5$

Suppose, that we can derive perturbed phase $x_{f}$ derived from phase equation (3).Since, $x_{f}$ is derivable, it can be as many times substitutable. There substituting $x_{f}$ back into the right hand side of (3), and thus removing $x_{f}$ on right side, we get,

$$
x_{f}=x_{o}-C \sin \left(x_{o}+\theta-C \sin \left(x_{o}+\theta-C \sin \left(x_{o}+\theta+\ldots\right)\right)\right)
$$

Lets denote,

$$
S_{x_{o}, C, \theta}[]=-C \sin \left(x_{o}+\theta+[]\right)
$$


then

$$
x_{f}=x_{o}+S_{x o, C, \theta}\left[S_{x o, C, \theta}[\ldots]\right]
$$

Suppose, in order to approximate $x_{f}$, we could replace nth recursion $S_{x_{o}, C, \theta}[\ldots]_{n}$ by a constant ' $k$ ',then as 'n' approaches infinity,then,

$$
\begin{gathered}
\lim _{n \rightarrow \infty}\left(\left[x_{f}\right]_{n}-\left[x_{f}\right]_{n+1}\right) \\
\lim _{n \rightarrow \infty}(k)=\lim _{n \rightarrow \infty}\left(-C \sin \left(x_{o}+\theta+\left[S_{x o, C, \theta}[\ldots]\right]_{n+2}\right)\right)
\end{gathered}
$$

since,

$$
x=A * \sin (x+t) \ldots . \forall t \neq 0
$$

has no solutions for $\mathrm{x}$, therefore (12) is invalid and (8) does not converge to $x_{f}$. For purpose of demonstration of result, note that,

$$
0 \leq\left|S_{x o, C, \theta}[]\right| \leq C
$$

therefore,for finite recursion, we can just replace $S_{x o, C, \theta}[]$ by $C+02=C 2$. On simulating the results, as shown in figure (3),simulated perturbed and theoretical perturbed phase do not appear same at all because the error between successive recursive operations appear same.Thus (8) is an extraneous solution and assumption of deriving it is wrong. Therefore,the assumption that we know $x_{f}$ for given $x_{o}$ is wrong.

5.3 Relationship of perturbed and unperturbed phase in frequency domain

We are going to use $\operatorname{norm}\left(L_{2}\right)$,in order to exploit the norm-modulus relationship,

$$
\epsilon=-C \sin \left(x_{f}+\arctan (\alpha)\right)
$$

Taking Fourier Transform on both sides and letting $\theta=\arctan (\alpha)$

$$
\begin{aligned}
& F[\epsilon]=-C F[\sin (x f+\arctan (\alpha))] \\
& F[\epsilon]=-C \sum_{k \geq 0}(-1)^{k} \frac{F\left[\left(x_{f}+\theta\right)^{2 k+1}\right]}{(2 k+1) !}
\end{aligned}
$$

Using Convolution theorem,

$$
F[\epsilon]=-C \sum_{k \geq 0}(-1)^{k} \frac{*_{1}^{2 k+1} F\left[\left(x_{f}+\theta\right)\right]}{(2 k+1) !}
$$

Taking norm $\left(L_{2}\right)$ on both sides,

$$
\begin{gathered}
\|F[\epsilon]\|=C\left\|\sum_{k \geq 0}(-1)^{k} \frac{*_{1}^{2 k+1} F\left[\left(x_{f}+\theta\right)\right]}{(2 k+1) !}\right\| \\
\|F[\epsilon]\|=C\left\|\sum_{k \in \text { even }} \frac{*_{1}^{2 k+1} F\left[\left(x_{f}+\theta\right)\right]}{(2 k+1) !}-\sum_{k \in \text { odd }} \frac{*_{1}^{2 k+1} F\left[\left(x_{f}+\theta\right)\right]}{(2 k+1) !}\right\|
\end{gathered}
$$

Under frame of triangular inequality

$$
\|a\|-\|b\| \leq\|a+b\| \leq\|a\|+\|b\|
$$


and Riesz-Throin theorem[5],for $\mathrm{p} \geq 1$,

$$
\begin{aligned}
& \|f * f\|_{p} \leq\|f\|_{1} \cdot\|f\|_{p} \\
& \|f * f\|_{2} \leq\|f\|_{1} \cdot\|f\|_{1}
\end{aligned}
$$

which implies,

$$
\left\|*_{1}^{2 k+1} f\right\|_{2} \leq\left(\|f\|_{1}\right)^{2 k+1}
$$

Representing, \|\|$_{2}$ as \|\| ,

$$
\begin{gathered}
\|F[\epsilon]\|=C\left\|\sum_{k \in \text { even }} \frac{*_{1}^{2 k+1} F\left[\left(x_{f}+\theta\right)\right]}{(2 k+1) !}-\sum_{k \in \text { odd }} \frac{*_{1}^{2 k+1} F\left[\left(x_{f}+\theta\right)\right]}{(2 k+1) !}\right\| \longrightarrow(a) \\
\|F[\epsilon]\| \leq C\left\|\sum_{k \in \text { even }} \frac{*_{1}^{2 k+1} F\left[\left(x_{f}+\theta\right)\right]}{(2 k+1) !}\right\|+C\left\|\sum_{k \in \text { odd }} \frac{*_{1}^{2 k+1} F\left[\left(x_{f}+\theta\right)\right]}{(2 k+1) !}\right\| \\
\|F[\epsilon]\| \leq C \sum_{k \in \text { even }} \frac{\left\|*_{1}^{2 k+1} F\left[\left(x_{f}+\theta\right)\right]\right\|}{(2 k+1) !}+C \sum_{k \in \text { odd }} \frac{\left\|*_{1}^{2 k+1} F\left[\left(x_{f}+\theta\right)\right]\right\|}{(2 k+1) !} \\
\|F[\epsilon]\| \leq C \sum_{k \geq 0} \frac{\left(\left\|F\left[x_{f}+\theta\right]\right\|_{1}\right)^{2 k+1}}{(2 k+1) !}
\end{gathered}
$$

Since,

$$
\sinh (x)=\sum_{k>0} \frac{x^{2 k+1}}{(2 k+1) !}
$$

therefore,

$$
\|F[\epsilon]\| \leq C \sinh \left\|F\left[x_{f}+\theta\right]\right\|_{1}
$$

Using (25),

$$
\|F[\epsilon]\|=C\left\|\sum_{k \in \text { even }} \frac{*_{1}^{2 k+1} F\left[\left(x_{f}+\theta\right)\right]}{(2 k+1) !}-\sum_{k \in \text { odd }} \frac{*_{1}^{2 k+1} F\left[\left(x_{f}+\theta\right)\right]}{(2 k+1) !}\right\| \longrightarrow(a)
$$

Applying triangular inequality again,

$$
\|F[\epsilon]\| \geq C\left\|\sum_{k \in \text { even }} \frac{*_{1}^{2 k+1} F\left[\left(x_{f}+\theta\right)\right]}{(2 k+1) !}\right\|-C\left\|\sum_{k \in \text { odd }} \frac{*_{1}^{2 k+1} F\left[\left(x_{f}+\theta\right)\right]}{(2 k+1) !}\right\|
$$

If the second normed sum is increased the inequality will surely retain as the overall result will be lesser if the first normed sum is fixed.So manipulating second normed sum,

$$
C\left\|\sum_{k \in \text { odd }} \frac{*_{1}^{2 k+1} F\left[\left(x_{f}+\theta\right)\right]}{(2 k+1) !}\right\| \leq C \sum_{k \in \text { odd }} \frac{\left(\left\|F\left[x_{f}+\theta\right]\right\|_{1}\right)^{2 k+1}}{(2 k+1) !} \leq C \sum_{k \geq 0} \frac{\left(\left\|F\left[x_{f}+\theta\right]\right\|_{1}\right)^{2 k+1}}{(2 k+1) !}
$$

and,

$$
C \sum_{k \geq 0} \frac{\left(\left\|F\left[x_{f}+\theta\right]\right\|_{1}\right)^{2 k+1}}{(2 k+1) !}=C \cdot \sinh \left\|F\left[x_{f}+\theta\right]\right\|_{1}
$$

Let

$$
f\left(x_{f}, \theta\right)=\left\|\sum_{k \in \text { even }} \frac{*_{1}^{2 k+1} F\left[\left(x_{f}+\theta\right)\right]}{(2 k+1) !}\right\|
$$

by using again triangular inequalities,just by add and subtracting suitable terms for higher bound and truncating at $\mathrm{k}=0$ for lower bound,we can find

$$
\left\|F\left[x_{f}+\theta\right]\right\| \leq f\left(x_{f}, \theta\right) \leq \frac{\sin \left(\left\|F\left[x_{f}+\theta\right]\right\|_{1}\right)+\sinh \left(\left\|F\left[x_{f}+\theta\right]\right\|_{1}\right)}{2}
$$


Combining results,

$$
\|F[\epsilon]\| \geq C f\left(x_{f}, \theta\right)-C \sinh \left(\left\|F\left[x_{f}+\theta\right]\right\|_{1}\right)
$$

Combining (30),(36) and (37),

$$
C f\left(x_{f}, \theta\right)-C \sinh \left(\left\|F\left[x_{f}+\theta\right]\right\|_{1}\right) \leq\|F[\epsilon]\| \leq C \sinh \left(\left\|F\left[x_{f}+\theta\right]\right\|_{1}\right)
$$

where,

$$
\left\|F\left[x_{f}+\theta\right]\right\| \leq f\left(x_{f}, \theta\right) \leq \frac{\sin \left(\left\|F\left[x_{f}+\theta\right]\right\|_{1}\right)+\sinh \left(\left\|F\left[x_{f}+\theta\right]\right\|_{1}\right)}{2}
$$

Firstly, from equation(3), $x_{o}-x_{f}=C \sin \left(x_{f}+\theta\right)$ Since sine is bounded function, therefore, $x_{F}$ acts as a cover around $x_{o}$ with maximum variation of $C$.

Moreover,for the purpose of analysis, lets define $\mu_{\tau}(\omega)$,defined over $\omega \geq 0$ as

$$
\mu_{\tau}(\omega)=1 \text { iff } \omega \leq \tau, \text { otherwise } 0
$$

then,

$$
\begin{aligned}
F_{\tau}\left[x_{o}(t)\right] & =F\left[x_{o}(t)\right] \mu_{\tau}(\omega) \\
F_{\tau}\left[x_{f}(t)\right] & =F\left[x_{f}(t)\right] \mu_{\tau}(\omega) \\
F_{\tau}[\epsilon(t)] & =F[\epsilon(t)] \mu_{\tau}(\omega)
\end{aligned}
$$

Consider, $x_{o}(t)$ as sum of finite $\mathrm{N}$ sinusoids as,

$$
x_{o}(t)=\sum_{n \geq 0}^{N} a_{n} \cos \left(\omega_{n}(t)\right)+b_{n} \sin \left(\omega_{n}(t)\right)
$$

Taking absolute of Fourier transform,we get,

$\left|F\left[x_{o}(t)\right]\right|=\sqrt{\frac{\pi}{2}} \sum_{n \geq 0}^{N}\left(a_{n}+b_{n}\right)\left(\delta\left(\omega-\omega_{n}\right)+\delta\left(\omega-\omega_{n}\right)\right)+\left(a_{m}-b_{n}\right)\left(\delta\left(\omega-\omega_{n}\right)-\delta\left(\omega+\omega_{n}\right)\right)$

Please note such function forms an almost zero everywhere for domain $\omega \geq 0$. When $\tau$ is allowed to vary from left to right of interval, $\left\|F_{\tau}\left[x_{o}(t)\right]\right\|=$ $\int_{0}^{\tau}\left|F_{\tau}\left(x_{o}\right)\right|^{2}$ increases discontinuously but rate is extremely low due to almost zero every where behaviour. On the other hand,from (38), $\left\|F_{\tau}\left[x_{o}(t)\right]\right\|$ increases rapidly and continuously by virtue of $\sinh \left(\left\|F_{\tau}\left[x_{f}(t)\right]+\frac{\theta}{\iota \omega}\right\|\right)$. This also allows greater magnitudes along higher frequencies. Therefore, from above, one can make a plausible inference that, in frequency domain, $x_{f}$ acts as noisy version (coverage of magnitudes over more frequency domain) of $x_{o}$. This also gives indication that $\left|F\left(x_{f}-x_{o}\right)\right|$ has no information of $x_{o}$. This is only possible, if $F\left(x_{f}\right)$ act as modified version of $F\left(x_{o}\right)$, then $F\left(x_{f}-x_{o}\right)=F\left(x_{f}\right)-F\left(x_{o}\right)$ would algebraically cancel out $F\left(x_{o}\right)$,removing information of $x_{o}$ to some extent,in frequency domain.

$$
F\left[x_{f}\right](\omega)=F\left[x_{o}\right](\omega)+T(\omega)
$$

where, per our assumption, $F\left[x_{o}\right](\omega)$ is almost zero every where (with some non zero elements at finite frequencies) and but $T(\omega)$ forms a continuous function with coverage over larger frequency domain and $F$. Thus, the extraction 
of $x_{o}$ from $x_{f}$ in frequency domain, becomes a spectral filtering process followed by minimizing contribution of $T(\omega)$ superimposed on $F\left[x_{o}\right](\omega)$, due to which extracted unperturbed phase would be slighty defornmed by just spectral filtering. The later part can be achieved by following some perturbative or homotopical deformation methods such that dynamics of equation(1) become preserved, inorder to tackle the problem of superposition.It is to be noted that this is not simple as ordinary filtering process and error is not random and has structure.This approach has been used in TFSP method[10] to estimate $x_{o}$ from $x_{f}$ without knowledge of $\mathrm{C}$ and $\alpha$ by simply putting a low pass filter which is an incomplete procedure.

\section{Simulation Results}

In order to exploit the break of continuous mapping when going from perturbed phase to unperturbed phase,from (1), taking time derivative on both sides,

$$
\begin{gathered}
\frac{d x_{o}}{d t}=\frac{d x_{f}}{d t}\left[1+C \cos \left(x_{f}+\theta\right)\right] \\
\frac{d x_{o}}{d x_{f}}=\left[1+C \cos \left(x_{f}+\theta\right)\right]
\end{gathered}
$$

Ofcourse, left hand side of (4) is equal to the right hand side, if the derivative exists and if derivative exists then by chain rule $\frac{d x_{o}}{d x_{f}}$ also exists, and we can say that continuous mapping between $x_{f}$ and $x_{o}$ exits. On plotting the left and right hand sides, for a given SMI signal, it is obvious from figure(4) that equation (15) does not holds and thus there exists no closed from continuous function from $x_{o}$ to $x_{f}$.

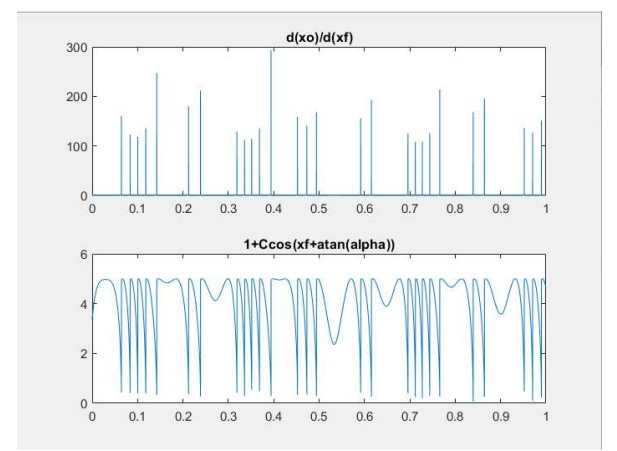

Fig. 4 Above plot represents left hand side of equation (15) and below plot represents right hand side of equation (15)

From Fourier norm manipulations, we deduce that the action of subtracting $x_{o}$ from $x_{f}$ removes information of $x_{o}$.It is obvious from figure (5), that Fourier transform of perturbed phase, while measurement range is in $\mu \mathrm{m}$, acts 
as modified over Fourier transform of unperturbed phase where new magnitudes are added where Fourier transform of unperturbed phase approaches zero, as shown in figure(5)

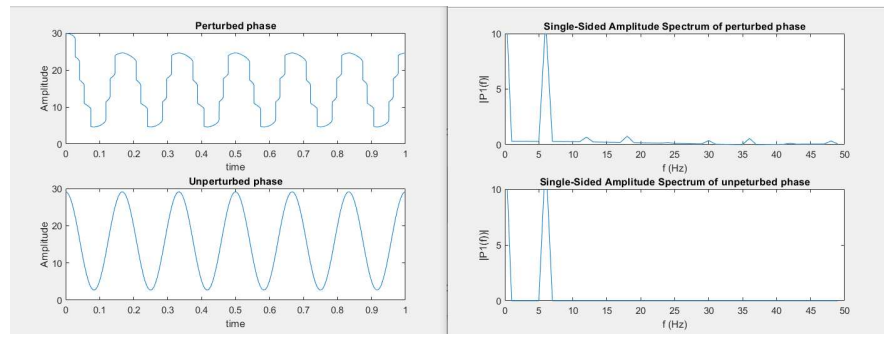

Fig. 5 left side represents unperturbed and perturbed phase for a $25 \mu \mathrm{m}$ sinusoidal $5 \mathrm{~Hz}$ displacement sampled at $10 \mathrm{kHz}$, right side represents their absolute Fourier transform in frequency domain

Therefore, a simple denoising process, as mentioned in TFSP method [10], carried out by an averaging filter[4] at 100 points, creates an error of less than $20 \mu \mathrm{m}$, which is quite not enough, since our range is in $\mu m$. This is shown in figure(6).

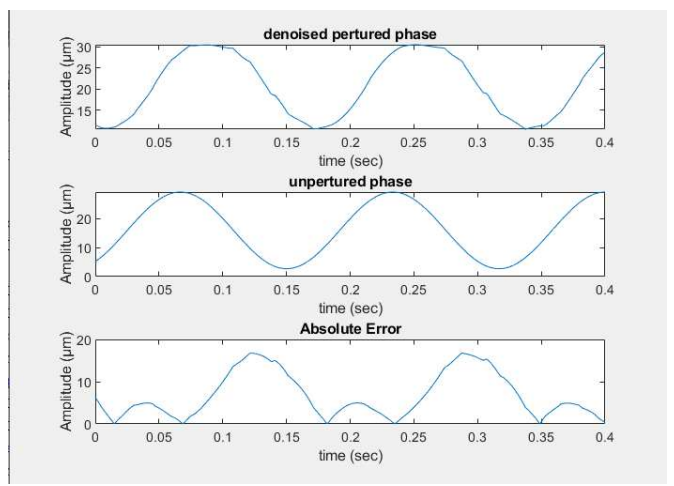

Fig. 6 Top to bottom represents denoised perturbed phase,unperturbed phase and absolute error

Another way to use approach of exploiting the noisy nature of perturbed phase is to use Savitzky-Golay filter[8] which is a smooting filter that tends to fit polynomials on subsets of adjacent data points. Given the structure of perturbed phase, as shown in figure (5) as triangular structures over unperturbed phase, makes Savitzky-Golay filter, the best method to exploit the TFSP method [10].As shown in figure (7),Savitzky-Golay filter is carried out, with frame length of 201 points, for a displacement of $5 \mathrm{~Hz}$ sampled at $10 \mathrm{kHz}$. Fig (7), shows that error between reconstructed phase $\left(x_{o r}\right)$ and $x_{o}$ is bounded 


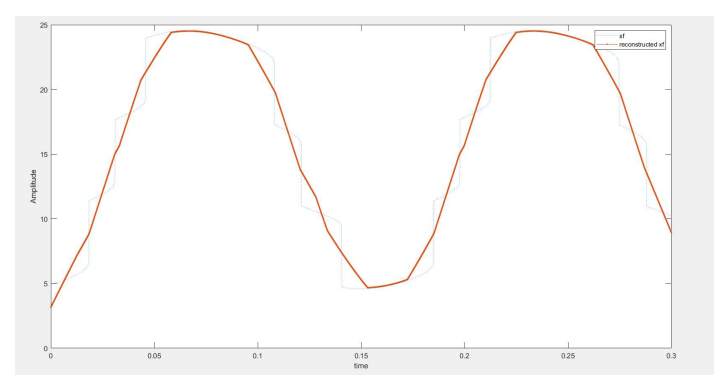

Fig. 7 dotted represents perturbed phase and bold represent reconstructed unperturbed phase by Savitzky-Golay filter

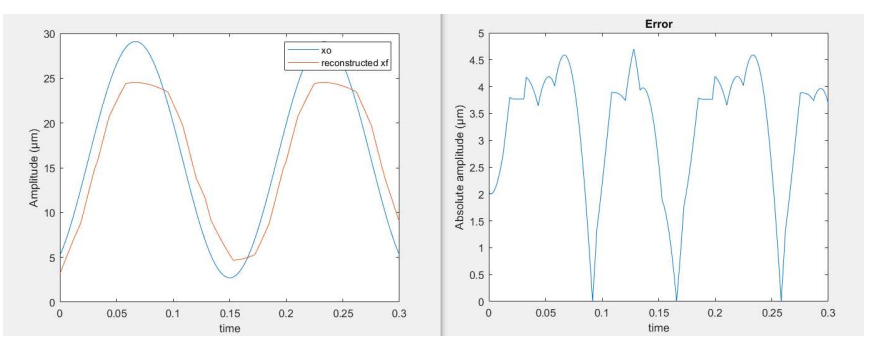

Fig. 8 Comparison of reconstructed unperturbed phase and unperturbed phase

by $4.5 \mu \mathrm{m}$. This is not enough since our range is already in $\mu \mathrm{m}$, while following just only filtering approach as mentioned in [10].

We can make important deduction from figure (7) that $x_{\text {or }}$ acts as homotopy of $x_{o}$, which can be continuously deformed onto one another,i.e, we can form a straight line homotopy between $x_{o r}$ and $x_{o}$ as,

$$
\zeta(s, t)=(1-s) x_{o r}(t)+s x_{o}(t)
$$

For purpose of analysis, lets assume $\alpha$ is known. Equation(41) is parameterised by $s \in[0,1]$ in such a way that when s moves from 0 to 1 , then

$$
C=\frac{x_{o r}-x_{f}}{\sin \left(x_{f}+\arctan (\alpha)\right)}
$$

C deforms from variable function to some constant function. Therefore, if it is possible to extract $x_{0}$ solely from $x_{f}$ knowledge, then it must be possible from homotopical or perturbation methods followed by spectral processing. This is not further explored in this paper as it goes the beyond the scope of purpose of this paper.

\section{Results and Discussion}

Since, perturbed phase $x_{f}$ cannot be represented as continuous differentiable function of unperturbed phase $x_{o}$, therefore methods for determination of displacement cannot be cannot involve interative calculus bases methods. For 
example, they cannot just rely on Taylor expansion to just estimate displacement from $x_{f}$ without going into knowledge of parameters $\mathrm{C}$ and $\alpha$. Moreover, the TFSP method approach [10] has been corrected and proven that moving from perturbed phase to displacement becomes spectral filtering in frequency domain, followed by perturbative or homotopical deformation in time domain.

\section{Conclusion}

It has been shown the impossibility to theoretically deriving $x_{f}$ from (1) as continuous function of $x_{o}$ through finite and infinite substitutions. Therefore we can never exactly derive displacement when estimating it just from knowledge of pertubred phase. Moreover, the nature of practical action of deriving $x_{o}$ from $x_{f}$ as it becomes a spectral filtering process followed by some perturbative or homotopical deformation.This provides target for any algorithm for robust estimation of displacement from estimated unperturbed phase $x_{o e}$ by $D(t)=$ $\frac{\lambda_{o} x_{o e}}{4 \pi}$ from recieved self mixing signal, when parameter $\mathrm{C}$ and $\alpha$ are uncertain.

\section{Declaration}

\subsection{Funding}

This study was not funded.

\subsection{Competing interests}

The author declares no conflicts of interest.

9.3 Availability of data and materials

Not applicable.

\subsection{Acknowledgements}

Simulation data was provided by Dr. Usman Zabit (National University of Sciences and Technology).

9.5 Author contribution

Not applicable 


\section{References}

1. Bernal, O.D., Zabit, U., Bosch, T.: Classification of laser self-mixing interferometric signal under moderate feedback. Applied optics 53(4), 702-708 (2014)

2. Bes, C., Plantier, G., Bosch, T.: Displacement measurements using a self-mixing laser diode under moderate feedback. IEEE transactions on instrumentation and measurement 55(4), 1101-1105 (2006)

3. Donati, S.: Developing self-mixing interferometry for instrumentation and measurements. Laser \& Photonics Reviews 6(3), 393-417 (2012)

4. Golestan, S., Ramezani, M., Guerrero, J.M., Freijedo, F.D., Monfared, M.: Moving average filter based phase-locked loops: Performance analysis and design guidelines. IEEE Transactions on Power Electronics 29(6), 2750-2763 (2013)

5. Krepela, M.: Convolution inequalities in weighted lorentz spaces. Math. Inequal. Appl $\mathbf{1 7}(4), 1201-1223$ (2014)

6. Lang, R., Kobayashi, K.: External optical feedback effects on semiconductor injection laser properties. IEEE journal of Quantum Electronics 16(3), 347-355 (1980)

7. Plantier, G., Bes, C., Bosch, T.: Behavioral model of a self-mixing laser diode sensor. IEEE Journal of Quantum Electronics 41(9), 1157-1167 (2005)

8. Schafer, R.W.: What is a savitzky-golay filter?[lecture notes]. IEEE Signal processing magazine 28(4), 111-117 (2011)

9. Taimre, T., Nikolić, M., Bertling, K., Lim, Y.L., Bosch, T., Rakić, A.D.: Laser feedback interferometry: a tutorial on the self-mixing effect for coherent sensing. Advances in Optics and Photonics 7(3), 570-631 (2015)

10. Zabit, U., Bernal, O.D., Amin, S., Qureshi, M.F., Khawaja, A.H., Bosch, T.: Spectral processing of self-mixing interferometric signal phase for improved vibration sensing under weak-and moderate-feedback regime. IEEE Sensors Journal 19(23), 11151-11158 (2019) 